\title{
Simultaneous detection of mRNA and protein in single cells using immunofluorescence-combined single-molecule RNA FISH
}

\author{
Jakub Kochan*, Mateusz Wawro*, and Aneta Kasza \\ Department of Cell Biochemistry, Faculty of Biochemistry, Biophysics, and Biotechnology, \\ Jagiellonian University, Cracow, Poland \\ *J.K. and M.W. contributed equally to this work
}

BioTechniques 59:209-221 (October 2015) doi 10.2144/000114340

Keywords: immunofluorescence; smRNA FISH; RNA; single-cell analysis; MCPIP1

Supplementary material for this article is available at www.BioTechniques.com/article/114340.

Although the concept of combining immunofluorescence (IF) with single-molecule RNA fluorescence in situ hybridization (smRNA FISH) seems obvious, the specific materials used during IF and smRNA FISH make it difficult to perform these procedures simultaneously on the same specimen. Even though there are reports where IF and smRNA FISH were combined with success, these were insufficient in terms of signal intensities, staining patterns, and GFP-compatibility, and a detailed exploration of the various factors that influence IF and smRNA FISH outcome has not been published yet. Here, we report a detailed study of conditions and reagents used in classic IF and smRNA FISH that allowed us to establish an easy, robust, and GFP-compatible procedure. Our protocol enables simultaneous detection of mRNA and protein quantity as well as the subcellular distribution of these molecules in single cells by combining an RNase-free modification of the IF technique and the more recent smRNA FISH method. Using this procedure, we have shown the direct interaction of RNase MCPIP1 with IL-6 mRNA. We also demonstrate the use of our protocol in heterogeneous cell population analysis, revealing cell-to-cell differences in mRNA and protein content.

Studies of gene expression often require accurate quantification of the mRNA of interest. In recent years, measurement of mRNA abundance has been based mostly on the use of real-time PCR, which allows for fast, accurate, and reliable quantification of the target transcript. Although precise, real-time PCR results represent the average expression of a specific mRNA in a cell population. Since cell-to-cell differences in mRNA level may be significant, and both the presence of an mRNA in particular cells and its intracellular localization are of crucial importance, novel methods allowing for quantification and subcellular localization of transcripts in single cells have been developed. One such method for imaging individual mRNA molecules in fixed cells is smRNA FISH (single-molecule RNA fluorescence in situ hybridization) followed by microscopic analysis (1, 2). smRNA FISH reveals RNA using transcript-specific probes consisting of complementary DNA oligonucleotides attached to a fluorescent dye. Owing to the simplicity of the chemistries involved, smRNA FISH does not require any genetic modifications or transfections, in contrast to the PP7 and MS2 systems (3), and is straightforward and easy to implement.
Although smRNA FISH produces precise and quantitative results, it is deficient in terms of providing information about mRNA-protein interactions. However, such data can be acquired by combining smRNA FISH with immunofluorescence (IF). Though the concept is simple, the existing protocols and materials used for IF and smRNA FISH make these methods difficult to combine and may lead to artifacts and/or failure of experiments.

To circumvent these problems, we performed detailed studies of the conditions and reagents used in classic IF and smRNA FISH protocols. We identified

\section{METHOD SUMMARY}

Here we present the development and optimization of a simple, robust, and GFP-compatible method enabling simultaneous protein and mRNA detection using immunofluorescence (IF) and single-molecule RNA fluorescence in situ hybridization (smRNA FISH). Our protocol should be applicable to every working IF procedure. 
factors that led to failure of simultaneous protein and RNA detection and optimized both the conditions and the order of steps to ensure success. Here we describe a procedure that combines RNase-free modifications of the IF technique with the smRNA FISH method based on the use of Stellaris probes.

\section{Materials and methods}

Cell culture

HeLa (human cervix adenocarcinoma)

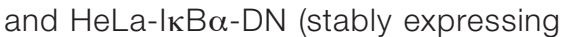
modified Addgene plasmid \#12329 coding for an undegradable form of I $\mathrm{B} \alpha$ protein, a gift from Inder Verman) cell lines were cultured in DMEM with $1.0 \mathrm{~g} / \mathrm{L}$ D-glucose (Lonza, Verviers, Belgium) supplemented with $2 \mathrm{mM}$ L-glutamine (Sigma-Aldrich, St. Louis, $\mathrm{MO}$ ) and $10 \%$ fetal bovine serum (FBS) (heat inactivated, South America origin, Biowest, Nuaillé, France). Medium for HeLa- $\left.\right|_{\kappa} B \alpha-D N$ cells was additionally supplemented with $5 \mu \mathrm{g} / \mathrm{mL}$ blasticidin (InvivoGen, San Diego, CA) as a selective antibiotic. TrypLE Express (Life
Technologies, Grand Island, NY) was used as a cell-dissociation agent. Cells were maintained at $37^{\circ} \mathrm{C}$ in humidified atmosphere with $5 \% \mathrm{CO}_{2}$. If applied, cytokines [recombinant human interleukin $1 \beta$ (IL-1 $\beta$ ) or recombinant human tumor necrosis factor (TNF); PromoKine, Heidelberg, Germany] were used at a concentration of $10 \mathrm{ng} / \mathrm{mL}$. For imaging experiments, cells were plated on glass coverslips (Waldemar Knittel Glasbearbeitungs, Braunschweig, Germany) in 12-well culture plates at a density of 60,000 cells/well (or 30,000 HeLa cells plus $30,000 \mathrm{HeLa-I \kappa B} \alpha-\mathrm{DN}$ cells for co-culture experiments) in $1 \mathrm{~mL}$ medium $24 \mathrm{~h}$ before treatment. For transfections, cells were plated in 12-well plates (Western blot analysis; 100,000 cells/well in $1 \mathrm{~mL}$ medium) or 24-well plates (Luciferase reporter gene assay; 100,000 cells/well in 0.5 $\mathrm{mL}$ medium) $24 \mathrm{~h}$ before transfection. All cell culture plasticware was purchased from BD Falcon (Corning Incorporated, Corning, NY). Cells were counted using a TC20 automated cell counter (Bio-Rad Laboratories, Hercules, CA).

\section{Molecular cloning}

Preparation of the plasmid constructs used in this report is described in the Supplementary Material.

\section{Quantitation of mRNA levels using} real-time quantitative PCR (qPCR)

Total RNA was isolated from cultured cells according to the protocol developed by Chomczynski and Sacchi (4). After isolation, RNA quality was assessed using agarose gel electrophoresis under denaturing conditions (5). RNA quantity was measured using a NanoDrop spectrophotometer (Thermo Fisher Scientific, Wilmington, DE), and equal amounts (1 $\mu \mathrm{g})$ were used in the reverse transcriptase (M-MLV Reverse Transcriptase; Promega Corporation, Madison, WI) reaction to generate cDNA. For qPCR, DNA fragments were amplified using the Kapa SYBR Fast qPCR Kit (Kapa Biosystems, Wilmington, MA), and primers specific to the transcripts being assessed [MCPIP1 and EEF2 (reference gene)]. Fluorescence was detected using the StepOnePlus Real-Time PCR System (Applied Biosystems, Waltham, MA). The MCPIP1
A
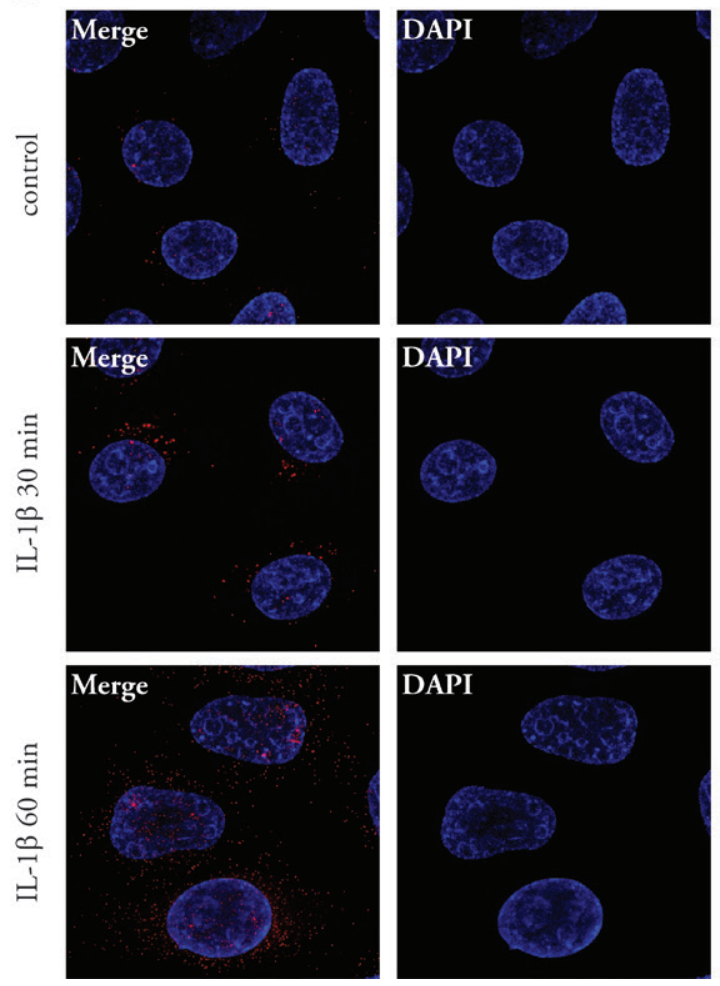
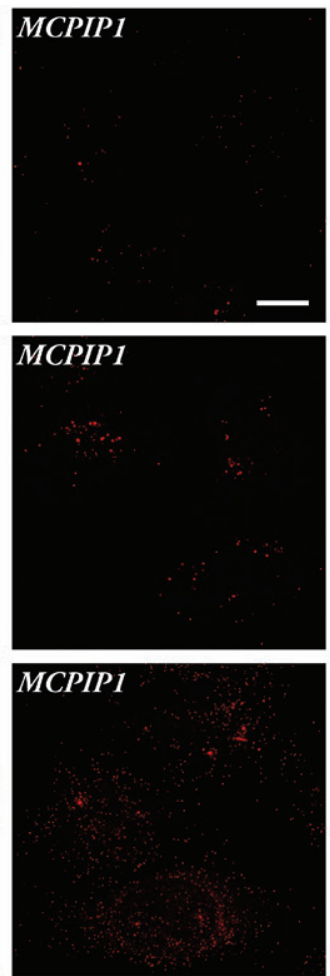

B
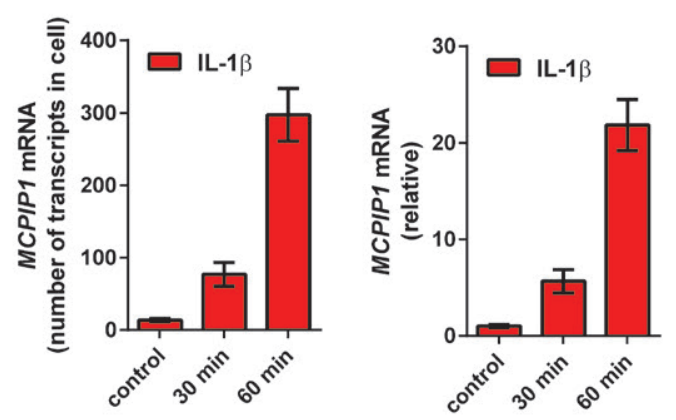

C

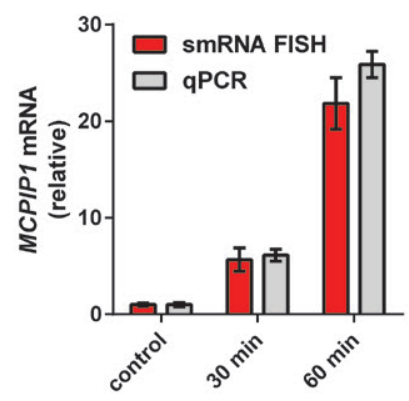

Figure 1. Quantification of MCPIP1 mRNA using single-molecule RNA fluorescence in situ hybridization (smRNA FISH). (A) Representative fluorescence images of HeLa cells stimulated with interleukin $1 \beta$ (IL-1 $\beta$ ) for 30 or 60 min and control cells (DAPI - nuclei, MCPIP1 - MCPIP1 mRNA detected using probe blend labeled with fluorescent dye Quasar 570). (B) Quantitative analysis of MCPIP1 mRNA. Left graph-number of transcripts in cell, right graph-relative amount of MCPIP1 mRNA based on smRNA FISH analysis. (C) Comparison of results obtained by use of smRNA FISH and real-time quantitative PCR (qPCR). Error bars represent mean \pm SEM (smRNA FISH, $n \geq 35)$ or SD (qPCR, $n=3$ ). Scale bar: $10 \mu m$. 
mRNA level in each sample was analyzed in duplicate, and the expression level was normalized to EEF2. The $\Delta \Delta \mathrm{Cq}$ method was used to calculate the final results (6). Primers used in qPCR (listed in Supplementary Table S2) were obtained from Genomed, Warszawa, Poland. Agarose gel electrophoresis and melting curve analysis confirmed production of single products. PCR efficiency was evaluated by serial dilution in duplicate. The efficiency was calculated as $10^{(-1 / \text { slope })}-1$. Efficiencies were $100 \%$ and $94 \%$ for MCPIP1 and EEF2, respectively (Supplementary Figure S1 and Supplementary Table S3).

\section{Preparation of cells for imaging}

For all imaging procedures cells, were plated on glass coverslips as described above. Basic precautions for minimizing RNase contamination were taken throughout all procedures. After treatment, cell culture medium was aspirated and cells were rinsed 2 times with $1 \times$ RNasefree PBS before being fixed for $10 \mathrm{~min}$ in 4\% methanol-free formaldehyde (Thermo Scientific, Rockford, IL) in 1× RNasefree PBS at room temperature. Fixative was aspirated, and cells were washed 3 times in 1x RNase-free PBS for 5 min each. Finally, all samples were mounted onto slides $(26 \mathrm{~mm} \times 76 \mathrm{~mm}$, MenzelGlaser/Thermo Scientific, Braunschweig, Germany) in VECTASHIELD Mounting Medium with DAPI to counterstain nuclei, (Vector Laboratories, Burlingame, CA), sealed with nail polish, and imaged as described in the "Widefield fluorescence microscopy" section.

Single-molecule RNA fluorescence in situ hybridization (smRNA FISH)

For smRNA FISH experiments alone, the procedure was carried out according to the manufacturer's instructions (Stellaris FISH protocol-Adherent cells; Biosearch Technologies, Inc., Petaluma, CA). The following probe blends (labeled with Quasar 570 dye) were used in this study: human GAPDH (125 nM), human MCPIP1 (250 nM), and human IL6 (1 $\mu \mathrm{M})$. Sequences of custom probe sets are listed in Supplementary Table S4. All hybridizations were done overnight in the dark at $37^{\circ} \mathrm{C}$ in a humidifying chamber.

Immunofluorescence (IF)

For IF experiments alone, a standard protocol was used. In brief, specimens were blocked and permeabilized for $60 \mathrm{~min}$ at room temperature in blocking buffer [1x PBS (Lonza), 5\% FBS, 0.3\% Triton X-100 (Sigma-Aldrich)]. Directly after blocking, cells were incubated with antibodies diluted in antibody dilution buffer [1x PBS, 1\% BSA (BioShop, Burlington, ON, Canada), 0.3\% Triton X-100]. EDC4/Ge-1 was stained with anti-EDC4/Ge-1 rabbit polyclonal antibody (1:400) (\#2548; Cell Signaling Technology, Danvers, MA) and Alexa Fluor 488-conjugated goat antirabbit antibody (1:150) (\#111-546-045; Jackson ImmunoResearch, Suffolk, UK).

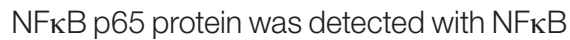
p65 Antibody (C-20) X (1:1000) (\#sc-372X; Santa Cruz Biotechnology, Dallas, TX) and Alexa Fluor 488-conjugated goat antirabbit antibody (1:150) (\#111-546-045; Jackson ImmunoResearch). MCPIP1MycHis and EGFP-MycHis proteins were stained with anti-c-myc mouse monoclonal antibody (1:200) (clone 9E10, MA1-980; Thermo Scientific, Rockford, IL) and Alexa Fluor 488-conjugated donkey anti-mouse antibody (1:150) (\#715-546-150, Jackson ImmunoResearch) or DyLight 549-conjugated rabbit anti-mouse antibody (1:150) (\#315-506-003, Jackson ImmunoResearch). All incubations with primary antibodies were done overnight in the dark at $4^{\circ} \mathrm{C}$, and incubations with secondary antibodies were done for 90 min in the dark at room temperature, in a humidifying chamber. In between incubations with antibodies and after incubation with secondary antibodies, cells were washed 3 times in $1 \times$ PBS for 5 min each.

\section{Combined IF/smRNA FISH}

A detailed protocol describing the procedure for Combined IF/smRNA FISH including all of the reagents, recipes and hints is provided in the Supplementary Material.

\section{Fluorescence microscopy}

All images were acquired using a fully motorized Leica DMI6000B (AF7000 version) inverted widefield fluorescence microscope (Leica Microsystems, Wetzlar, Germany). Images were recorded with a high numerical aperture 100x oil immersion objective (HCX PL APO 100.0 $\times 1.47$ oil; Leica Microsystems) [immersion oil, Nikon 50 Type $A, n_{D}$ (refractive index) = 1.515 (at $23^{\circ} \mathrm{C}$ ); Nikon Instruments Europe BV, Amsterdam, The Netherlands] using a 14-bit Hamamatsu 9100-02 EM-CCD

\section{The Superior Antifade}

\section{VECTASHIELD ${ }^{\circledR}$ Mounting Medium}

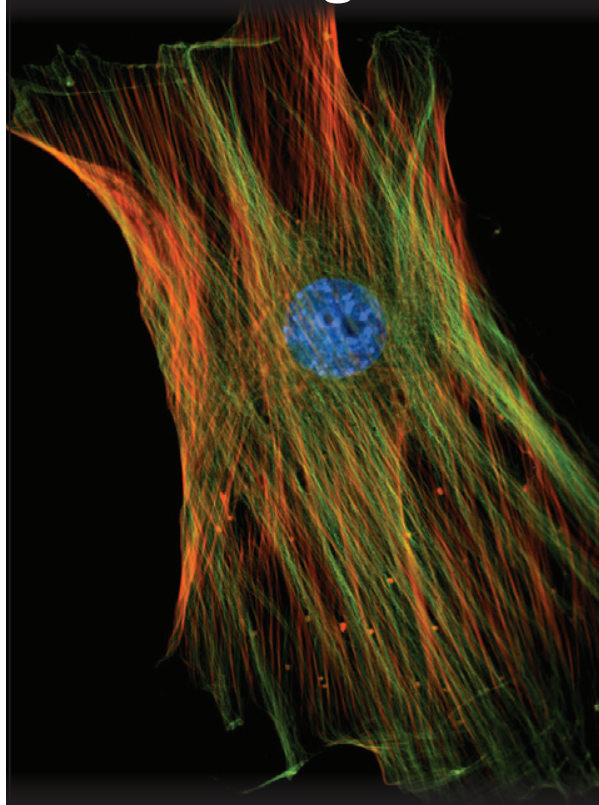

- Inhibits fading of dyes and fluorescent proteins

- Ideal refractive index

- Ready to use

- Offered with nuclear or cytoskeletal counterstains

- Hardening or non-hardening formulations

- Can be stored without sealing for long term analysis

\section{FREE sample offer!}

To learn more go to www.vectorlabs.com/vstrial-bt

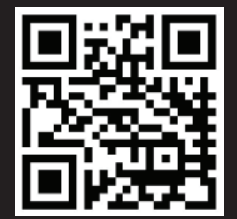


High Speed Set cooled CCD camera (Hamamatsu Photonics, Hamamatsu, Japan) with Leica Application Suite Advanced Fluorescence image acquisition software. The following filter sets (Leica Microsystems) were used: A4 for detection of DAPI, GFP-T ET for detection of Alexa Fluor 488 Dye and GFP, N2.1 for detection of DyLight 549 Dye, and Rhod ET for detection of Quasar 570 Dye. After deconvolution from 40-50 z-sections with 0.2 um spacing, images were analyzed by local background subtraction and thresholding using Huygens Software (Scientific Volume Imaging, SVI, Hilversum, The Netherlands). The number of cytoplasmic mRNA transcripts per cell was approximated by totaling the number of mRNA foci divided by the number of complete nuclei in each image. Final image adjustments were done using ImageJ 1.48v (National Institutes of Health, Bethesda, MD), Adobe Photoshop CS4 Extended Version 11.0.2, and Adobe Illustrator CS4 Version 14.0.0 (Adobe Systems, San Jose, CA).

\section{Transfections}

HeLa cells were transfected with plasmid DNA using Lipofectamine 2000 reagent (Invitrogen, Carlsbad, CA) according to the manufacturer's instructions.

\section{Luciferase reporter gene assay}

A total of $0.8 \mu \mathrm{g}$ DNA per well was used, including $0.7 \mu \mathrm{g}$ of pmirGLO dual luciferase expression vector (pmirGLO-IL6-UTR, pmirGLO-IL-6-UTRACE, or empty pmirGLO; Promega Corporation) and 25 ng of MCPIP1-MycHis expression vector. The amount of DNA per well was equalized to $0.8 \mu \mathrm{g}$ with mock DNA (pcDNA3.1/ MycHisA). Twenty-four hours after transfection, cells were lysed and assayed for luciferase activity using the Dual-Luciferase Reporter Assay System (Promega Corporation) according to the manufacturer's instructions. Renilla luciferase served as an internal control.

\section{Western blot analysis}

HeLa cells were transfected a 12-well plate with the MCPIP1-MycHis expression vector or an empty vector. Twentyfour hours after transfection, cells were lysed in RIPA buffer [25 mM Tris- $\mathrm{HCl} \mathrm{pH}$ 7.6 (BioShop), 150 mM NaCl (Avantor Performance Materials Poland, Gliwice, Poland), 1\% NP40 (Sigma-Aldrich), 1\% sodium deoxycholate (Sigma-Aldrich),
0.1\% SDS (Sigma-Aldrich)] supplemented with cOmplete ULTRA protease inhibitors (Roche, Mannheim, Germany) and $1 \mathrm{mM}$ PMSF (Sigma-Aldrich). Protein extracts $(20 \mu \mathrm{g})$ were separated by SDS-PAGE and wet-transferred onto an ImmobilonP PVDF membrane (Millipore Corporation, Billerica, MA). The membrane was blocked for $1 \mathrm{~h}$ at room temperature in 5\% w/v nonfat dry milk (BioShop) in TTBS [20 mM Tris, 150 mM NaCl, 0.1\% Tween-20 (SigmaAldrich)]. After blocking, membranes were incubated overnight with gentle agitation at $4^{\circ} \mathrm{C}$ with the following primary antibodies: anti-c-Myc (clone 9E10) or anti-GAPDH (Abcam, Cambridge, UK), washed 3 times for 5 min each with $25 \mathrm{~mL}$ TTBS and incubated with HRP-conjugated secondary antibodies (anti-mouse or antirabbit; Cell Signaling Technology) for $1 \mathrm{~h}$ at room temperature. All antibodies were diluted in blocking buffer. After incubation with secondary antibodies, membranes were washed 3 times for 5 min each with $25 \mathrm{~mL}$ TTBS, and the luminescence was detected using Immobilon Western Chemiluminescent HRP Substrate (Millipore Corporation) and recorded on X-ray film (Retina, Berlin, Germany).

\section{Results and discussion}

MCPIP1 (also known as Regnase-1) is a recently identified endonuclease that destabilizes a set of transcripts coding for cytokines playing a key role in the inflammatory response and immune homeostasis (7). We previously demonstrated that transcription factors Elk-1 and SRF were involved in IL1-dependent regulation of MCPIP1 expression (8). During our studies of MCPIP1 function and its interactions with target transcripts, the need for a method enabling simultaneous detection of mRNA and protein in single cells arose. Although some protocols for such procedures were available, we found it disappointing that none of them worked properly. Here we present detailed studies that led to the development of a simple and robust protocol for IF combined with smRNA FISH that enables simultaneous protein and $\mathrm{mRNA}$ detection at the singlecell level.

smRNA FISH results are similar to data obtained in qPCR

To address the issues of mRNA subcellular localization and association with partner proteins, we began by using a recently developed method for imaging individual mRNA molecules in fixed cells, smRNA FISH, using Stellaris probe blends. We used a custom-made blend of probes complementary to the MCPIP1 mRNA coding for the MCPIP1 protein according to the protocol supplied by the manufacturer. Although we expected that extensive optimization would be needed, almost none was required as the probe set and hybridization protocol supplied by the manufacturer allowed us to obtain highquality images (Figure 1A). To determine whether the signal produced by the probe set is not only qualitative but also quantitative, and to verify that the probes hybridize only with MCPIP1 mRNA and not with other RNAs, we decided to compare the data obtained from smRNA-FISH with gPCR data. HeLa cells were stimulated with IL-1 $\beta$ or TNF, cytokines known to highly (20-30 fold) induce expression of MCPIP1 mRNA (7), and then both smRNA-FISH and qPCR analyses were performed. As expected, IL-1 $\beta$ (Figure 1, $A$ and $B$ ) and TNF (Supplementary Figure S2, A and B) induced MCPIP1 expression as revealed by qPCR. smRNA-FISH images also revealed a robust increase in MCPIP1 mRNA quantity, with transcribed regions in the genome (bright spots in the nucleus) clearly visible. Quantification of the smRNA-FISH signal revealed that smRNA FISH is fully quantitative. The induction level of MCPIP1 mRNA upon cytokine treatment calculated from smRNA-FISH images is equivalent to data obtained by qPCR (Figure $1 \mathrm{C}$ and Supplementary Figure S2C). The quantitation of the smRNA-FISH signal also indicates that the signal comes only from MCPIP1 mRNA and not other RNAs. Thus, we concluded that smRNA-FISH allowed us to detect not only the spatial distribution of a specific transcript but also to quantitate it in single cells.

smRNA FISH protocol is not compatible with standard IF

Since we were interested in the simultaneous and direct detection of mRNA and protein in single cells, we investigated the compatibility of our smRNA FISH setup with IF. In these experiments, we used a pre-designed control probe blend to detect human GAPDH mRNA (Figure 2A, upper panel, and Figure 3C, panel 3). EDC4 (also known as Ge-1 or 
A
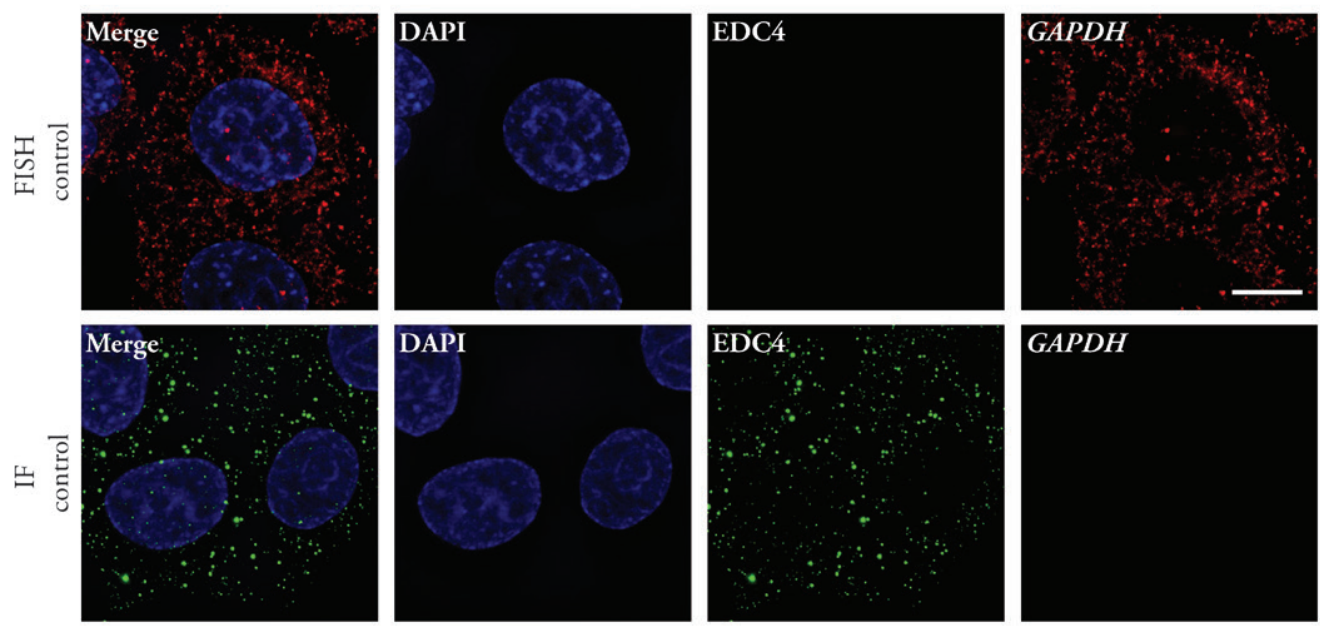

B
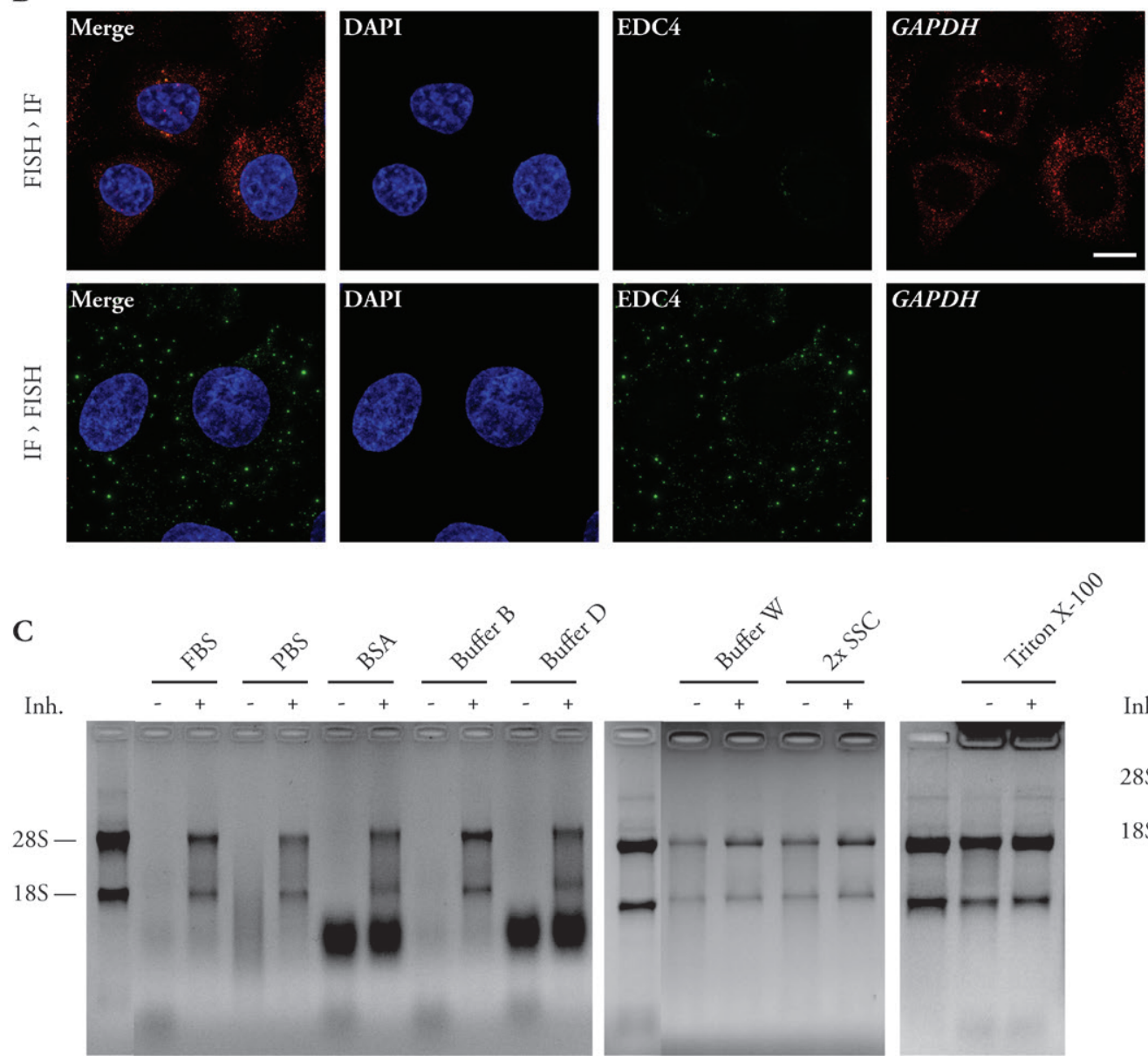

Inh.

Figure 2. Identification of key factors influencing the combined immunofluorescence/ single-molecule RNA fluorescence in situ hybridization (IF/ smRNA FISH) procedure. (A) Representative control fluorescence images of HeLa cells after smRNA FISH only or IF only (DAPI - nuclei; EDC4 - primary antibody: rabbit anti-EDC4/Ge-1, secondary antibody: Alexa Fluor 488-conjugated goat anti-rabbit; GAPDH - GAPDH mRNA detected using probe blend labeled with fluorescent dye Quasar 570). (B) Standard procedures do not allow for simultaneous detection of protein and mRNA. The variant procedure where smRNA FISH is followed by IF (FISH$>$ IF) and the reverse protocol (IF->FISH) were both inefficient. Representative fluorescence images of HeLa cells stained as in (A). (C) Identification of sources of RNases among reagents used in IF and smRNA FISH. All reagents were used at concentrations reflecting real conditions during analyzed procedures. Buffer B-blocking buffer, Buffer D-antibody dilution buffer, Buffer W-smRNA FISH wash buffer, Ac-BSA-acetylated BSA (RNase free), PBS(DEPC)-DEPC-treated PBS (RNase free). Inh.-RNase inhibitor (Protector RNase Inhibitor; Roche). Gels show results of denaturing agarose RNA electrophoresis, as assessed by the $28 \mathrm{~S}$ and $18 \mathrm{~S}$ rRNA bands. Scale bars for (A) and (B): $10 \mu \mathrm{m}$.
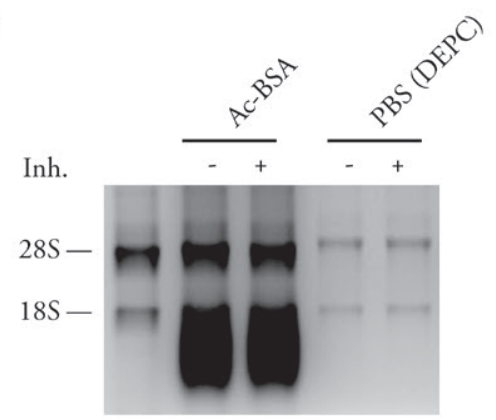

HEDLS) served as a target protein in IF (Figure 2A, lower panel, and Figure 3C, panel 6). EDC4 is an essential component of cytoplasmic P-bodies responsible for mRNA decapping and degradation (9). GAPDH mRNA detection through smRNA FISH and EDC4 IF alone both resulted in the expected stained images, with a dispersed cytoplasmic signal for
GAPDH and distinct cytoplasmic bright spots for EDC4 (Figure 2A). As we did not yet know how to properly combine smRNA FISH with IF, we decided initially to use two alternative procedures: In the first, the standard smRNA-FISH protocol was followed by the standard IF protocol (FISH->IF), and in the second, the reverse order was applied (IF->FISH). Surprisingly, both procedures resulted in failure of the second-step protocol (Figure 2B). When FISH was followed by IF, we were unable to detect EDC4 spots, while GAPDH was visible although the signal was much weaker when compared with FISH alone. When FISH followed IF, the EDC4 spots were clearly visible, and the signal was comparable (or weaker) to IF alone, but 
A

\begin{tabular}{|c|c|}
\hline $\mathrm{EtOH}$ & PBS \\
\hline 1 & 4 \\
\hline 2 & 5 \\
\hline$\underset{\substack{\text { smRNA FISH } \\
\text { (controll) }}}{3}$ & 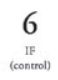 \\
\hline
\end{tabular}

C

1 Merge

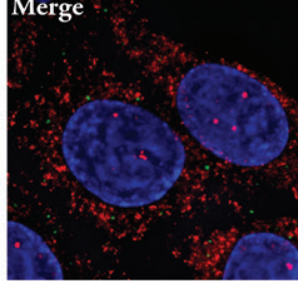

2 Merge

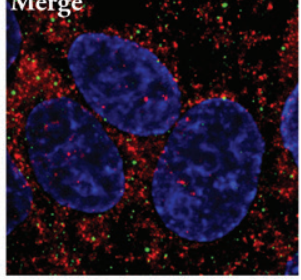

3 Merge

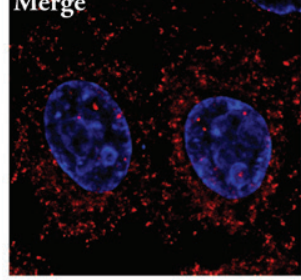

4 Merge

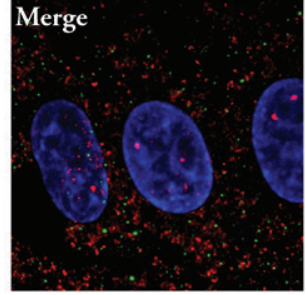

5 Merge

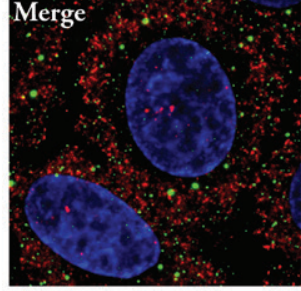

6 Merge

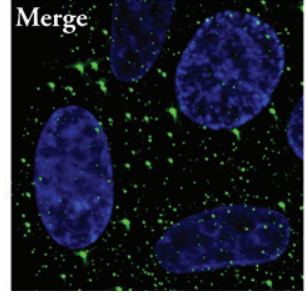

B

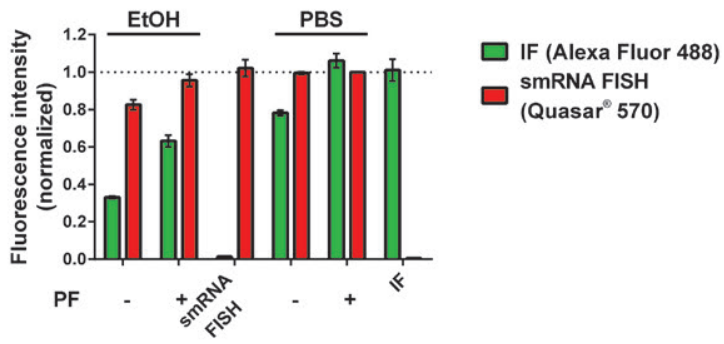

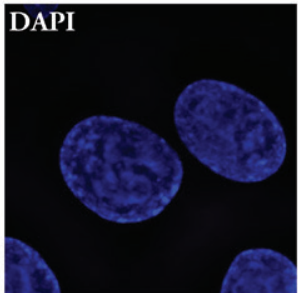

DAPI
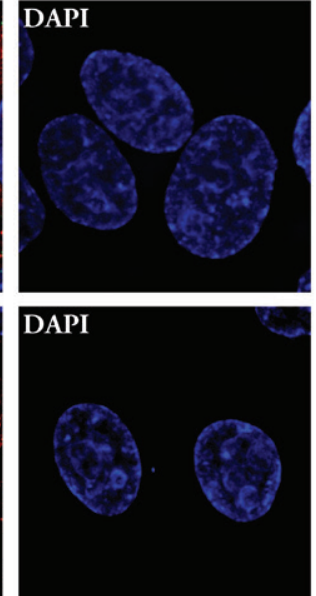

DAPI
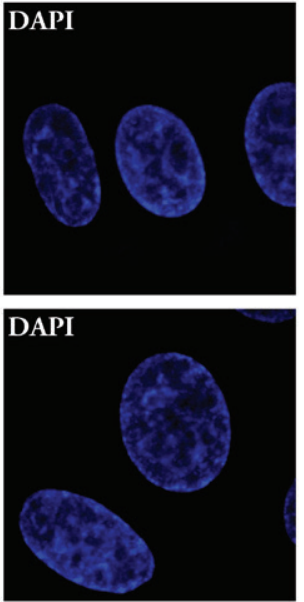

DAPI

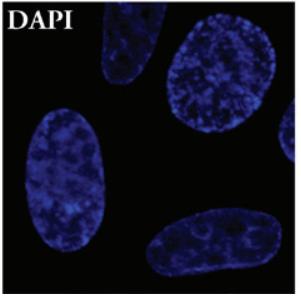

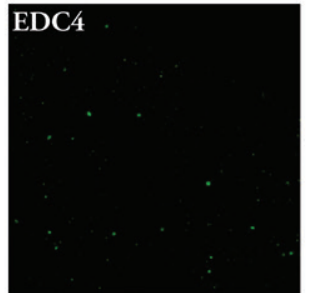

EDC4
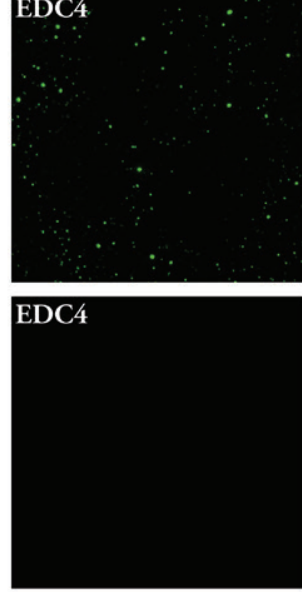

EDC4
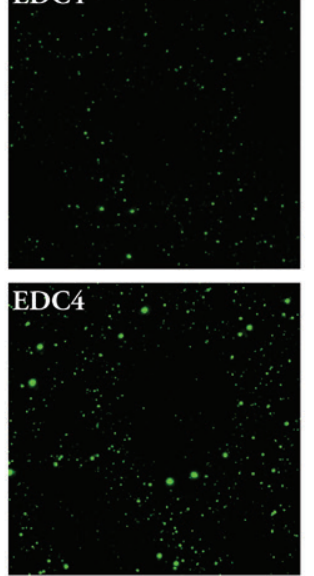

EDC4

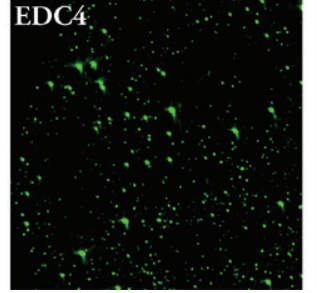

GAPDH

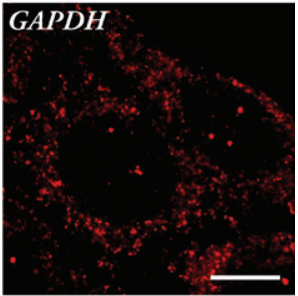

GAPDH
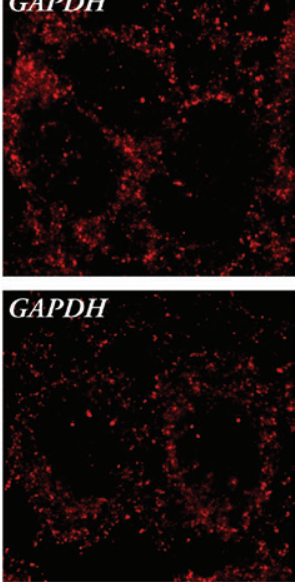

GAPDH

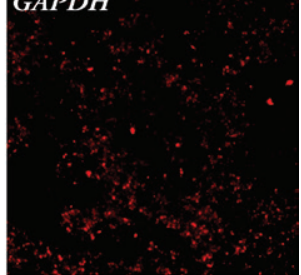

GAPDH

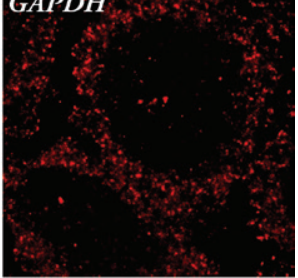

Figure 3. Optimization of procedure where protein detection by immunoflorescence (IF) is performed first and RNA is then detected by single-molecule RNA fluorescence in situ hybridization (smRNA FISH). (A) Schematic depicting subvariants presented in (C). (B) Graph showing normalized fluorescence intensities of images of tested subvariants, for each channel (IF - Alexa Fluor 488 Dye, green channel; smRNA FISH - Quasar 570, red channel). Measured fluorescence intensities of raw images of tested subvariants (mean values) were normalized to control images, where only IF or smRNA FISH were performed. Error bars represent mean \pm SEM $(n \geq 35)$. (C) Representative fluorescence images of HeLa cells after IF->FISH (DAPI - nuclei; EDC4 - primary antibody: rabbit anti-EDC4/Ge-1, secondary antibody: Alexa Fluor 488-conjugated goat antirabbit; GAPDH - GAPDH mRNA detected using probe blend labeled with fluorescent dye Quasar 570). Scale bar: $10 \mu \mathrm{m}$. 
no FISH signal could be detected. Even though we had assumed that the IF->FISH procedure might not work due to the fact that the materials used in IF are mostly not RNase-free, the failure of IF in the FISH->IF procedure was unexpected. It appeared that the FISH procedure changed the protein epitopes so that they were no longer recognized by the antibodies or that some of the residual reagents used in the FISH procedure were still present during IF and inhibited antibody-antigen interactions. The FISH procedure involves harsh conditions: high salt concentrations, elevated temperature, and formamidea reagent known to disrupt antibodyantigen interactions (10). The presence of formamide was assumed to be the main reason for the unsuccessful IF after FISH. A procedure based on antibodies added directly to hybridization buffer (primary antibodies) and wash buffer (secondary antibodies), which was proposed by the manufacturer of the smRNA FISH probe blends and reported by Shih et al. (11), also resulted in experimental failure. Moreover, the addition of secondary antibodies alone led to artifactual signals (Supplementary Figure $\mathrm{S} 3 \mathrm{H}$ and Supplementary Figure $\mathrm{S} 4 \mathrm{H})$. Since the use of extensive washes to remove salts and formamide after the FISH procedure and before IF did not help, we chose the IF->FISH procedure as a more promising variant for further development. Our choice of IF followed by FISH was further supported by the protocol proposed by Grünwald et al. (12), which we found partially successful but insufficient in terms of signal intensities, staining patterns, and the formation of secondary antibody artifactual speckles (discussed in detail below).

\section{Development of RNase-free IF protocol}

To understand the failure of the combination of IF and smRNA FISH, we performed extensive order-of-steps studies (Supplementary Figures S3 and S4). These included the standard IF protocol, the IF protocol without using Triton X-100 (we hypothesized that presence of detergent throughout all of the procedures might lead to RNA loss from the cytoplasm or affect the RNA-probe interaction), the IF protocol with Triton X-100 excluded from blocking or antibody dilution buffer, or the protocol without using Triton X-100 but with ethanol permeabilization. These IF trials were then followed by the standard smRNA FISH protocol. In all of these variants, incubation with primary antibodies was performed at $4^{\circ} \mathrm{C}$ and smRNA FISH was performed at $37^{\circ} \mathrm{C}$. We also tested incubation with primary antibodies at $37^{\circ} \mathrm{C}$ followed by smRNA FISH at $37^{\circ} \mathrm{C}$ and incubation with primary antibodies at $4^{\circ} \mathrm{C}$ followed by smRNA FISH at $4^{\circ} \mathrm{C}$. Unfortunately, none of these protocols allowed for simultaneous and direct detection of mRNA and proteins. Since we did not observe any correlation of smRNA FISH signal with the order of the steps or the temperature at which they were carried out (with the exception of the observation that hybridization cannot be done at $4^{\circ} \mathrm{C}$ and that antibodies cannot be added to smRNA FISH buffers), we decided to examine all of the reagents used in classic IF and smRNA FISH protocols for the presence of RNase activity. These tests revealed that while all reagents used in smRNA FISH are RNase-free, almost all reagents used in IF (except Triton $\mathrm{X}$-100) are contaminated with RNases (Figure 2C).

\section{Results of RNase-free IF protocol} followed by smRNA FISH

We reasoned that removing RNase contamination from IF reagents should allow the combining of IF with smRNA FISH. We removed FBS and BSA from all the buffers and substituted them with RNase-free, in-house acetylated BSA [prepared using the modified procedure described by Gonzalez et al. (13) and online procedure from the web resources of the Malaysian Cocoa Board (www. koko.gov.my); see protocol in the Supplementary Material]. RNases contaminating PBS were removed by DEPC treatment. The treated BSA and PBS were tested to ensure that they did not degrade RNA (Figure 2C, right-most panel). With these reagents in hand and with the knowledge obtained from our previous attempts, we decided to test and optimize a protocol variant where protein detection is performed first (RNase-free IF), and RNA is then detected (smRNA FISH). We decided also to include a post-fixation step after incubation with secondary antibodies but before starting hybridization in order to prevent the removal or displacement of antibodies. A summary of this final optimization is presented in Figure 3. Although all four of the tested protocol subvariants based on RNase-free, acetylated BSA as a blocking agent allowed for simultaneous detection of protein and mRNA, detailed analysis of the acquired images revealed significant differences in IF signal intensities (Figure 3B). Ethanol permeabilization resulted in a dramatic decrease in IF signal when compared with the control IF (33\%), while omitting ethanol permeabilization but blocking specimens with blocking buffer resulted in a huge increase in IF intensity (78\% of control). Post-fixation resulted in an even more pronounced increase of IF and smRNA FISH signals, reaching $106 \%$ and $100 \%$ of control specimen fluorescence intensities, respectively. As predicted, similar procedures utilizing FBS led to experiment failure (Supplementary Figure S5).

Having optimized our IF-combined smRNA FISH protocol, we decided to compare it with the one proposed by Grünwald et al. (12) using the same RNasefree regents we tested previously. As we expected from our in-optimization observations, images obtained from specimens stained as described by Grünwald et al. still produced much weaker signals, both in the IF and the smRNA FISH channels, when compared with our protocol and led to the formation of the aforementioned artifacts (Supplementary Figure S6). The reason for the better performance of our protocol in comparison to the one suggested by Grünwald et al. is due to differences in the order of steps and the reagents used. First, the order of steps presented by Grünwald et al. is primary antibody staining -> post-fixation -> smRNA-FISH -> secondary antibody staining. We found that this order is not optimal since, although the post-fixation step retains primary antibodies bound to epitopes, the formamide used in the subsequent smRNA FISH procedure greatly weakens binding of the secondary antibodies, even after extensive washing, resulting in weaker signal. Second, Grünwald et al. used alcohol permeabilization, which we also found to significantly reduce signal intensities in the IF channel. Moreover, alcohol permeabilization as well as the elevated hybridization temperatures suggested in another protocol by Toledano et al. (14) cause substantial loss of GFP fluorescence (Supplementary Figure S6, B and C) as also reported by Nakamura et al. (15). This loss of fluorescence is not observed in our protocol, making it the method of choice when preservation of GFP signal is of crucial importance. 

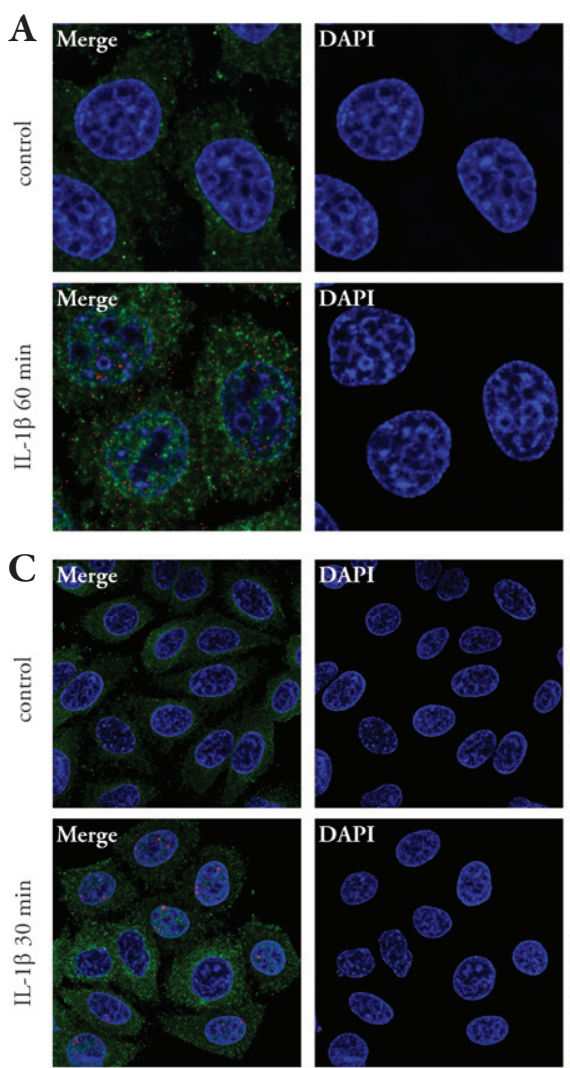

D

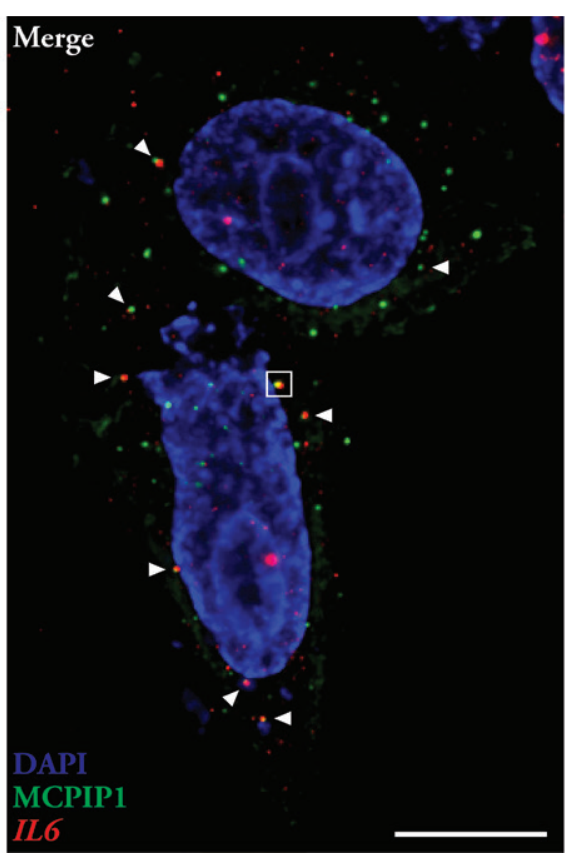

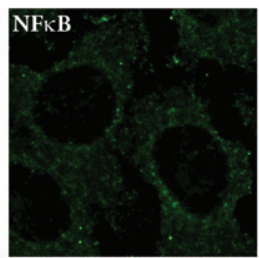
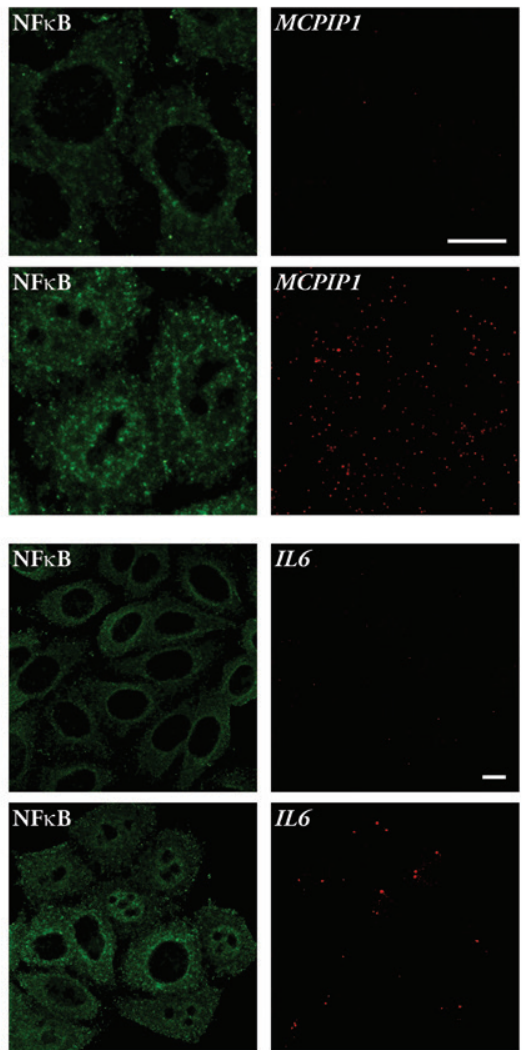

E

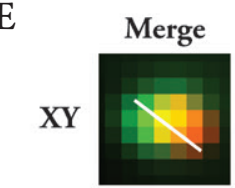

MCPIP1

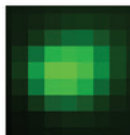

$\mathrm{XZ}$
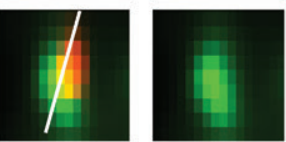

YZ
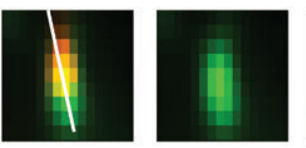

F

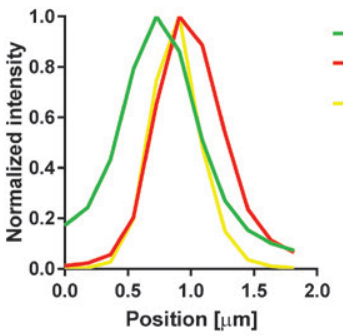

B

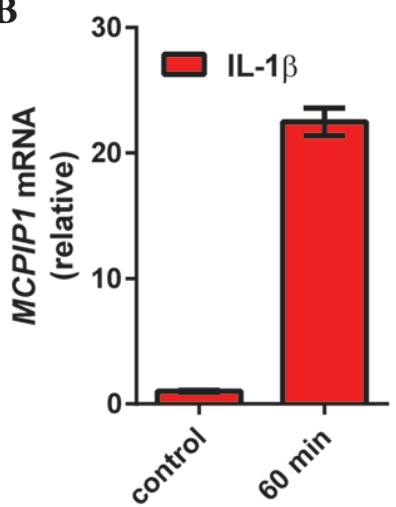

G

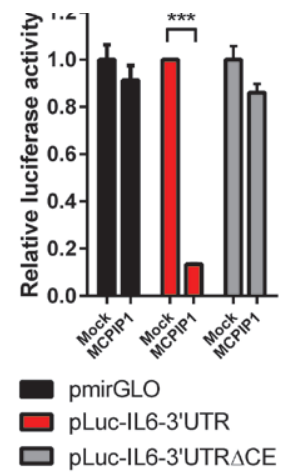

H

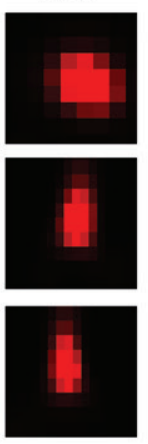

- MCPIP1

- IL6

Iso-colocalization surface

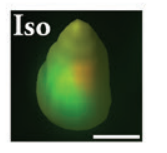

Mock MCPIP1

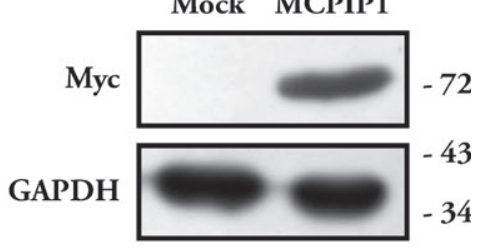

Figure 4. Verification of the simultaneous protein and RNA detection procedure. (A) Representative fluorescence images of HeLa cells stimulated with interleukin $1 \beta$ (IL-1 $\beta$ ) and control cells subjected to our IF->FISH procedure (DAPI - nuclei; NFkB - primary antibody: rabbit anti-NFkB p65, secondary antibody: Alexa Fluor 488-conjugated goat anti-rabbit; MCPIP1 - MCPIP1 mRNA detected using probe blend labeled with fluorescent dye Quasar 570). (B) Quantitative analysis of MCPIP1 mRNA_relative MCPIP1 mRNA level based on smRNA FISH data. Error bars represent mean \pm SEM (smRNA FISH, $n \geq$

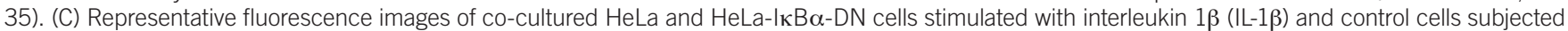

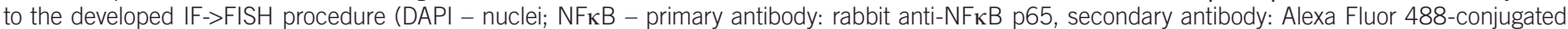
goat anti-rabbit; IL6 - IL6 mRNA detected using probe blend labeled with fluorescent dye Quasar 570). (D) Representative fluorescence image of HeLa cells transfected with MCPIP1 MycHis-tagged expression vector and stained for c-myc and IL6 mRNA (DAPI - nuclei; MCPIP1 - primary antibody: mouse anti-cmyc, secondary antibody: Alexa Fluor 488-conjugated donkey anti-mouse; IL6 - IL6 mRNA detected using probe blend labeled with fluorescent dye Quasar 570). White arrows indicate co-localization of MCPIP1 with IL6 mRNA. Boxed area is presented in more detail in (E). (F) An intensity line profile illustrating the MCPIP1/IL6 co-localization observed in (E). (G) Luciferase reporter gene assay results proving the functionality of ectopically expressed MCPIP1-MycHis protein. Error bars represent mean $\pm \mathrm{SD}(n=3)$; data were analyzed with one-way ANOVA with Dunnett's multiple comparison post test $\left({ }^{* *} P<0.001\right)(H)$ Western blot analysis of transfected cells showing expression of the MCPIP1-MycHis protein. Scale bars in (A), (C), and (D): $10 \mu \mathrm{m},(E): 1.78 \mu \mathrm{m},(\mathrm{F}): 0.5 \mu \mathrm{m}$. 
We make really good antibodies.

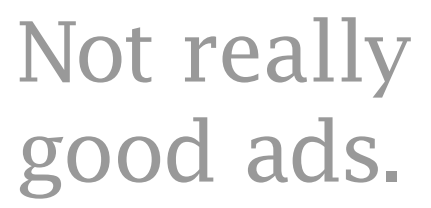

For really good antibodies, visit bethyl.com/trialsize

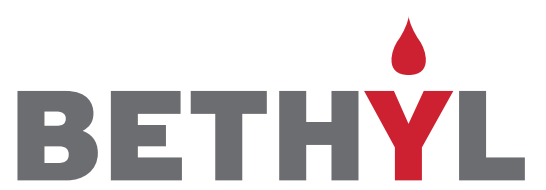

LA B O R A T O R I E S, I N C
To fully test the feasibility of our procedure, we imaged NFKB (p65) protein and MCPIP1 mRNA in HeLa cells stimulated with IL-1 $\beta$. In control cells, NF-kB is sequestered in the cytoplasm by $I_{\kappa} \mathrm{B}$ inhibitory proteins. Upon stimulation with $\mathrm{IL}-1 \beta$, I $\mathrm{KB}$ proteins are phosphorylated and targeted for rapid degradation through the ubiquitin-proteasome pathway, and the released NF- $\mathrm{KB}$ enters the nucleus where it regulates gene expression (16). IL-1 $\beta$-induced MCPIP1 gene expression is predominantly NF-кB dependent (17). Using our procedure, we could observe that MCPIP1 mRNA induction is accompanied by translocation of NF-kB from the cytoplasm to the nucleus of stimulated cells (Figure 4A). Quantitative analysis of MCPIP1 mRNA in this experiment (Figure 4B) corresponded well to the GPCR data presented above (Figure 1). In another example, we imaged NFKB (p65) protein and IL6 mRNA in co-cultured HeLa and HeLa-IкB $\alpha$-DN cells upon IL-1 $\beta$ stimulation. Translocation of NF- $\mathrm{\kappa B}$ and ILG mRNA induction were observed only in the unmodified cells. HeLa-IKB $\alpha$-DN cells express high level of modified, unphosphorylatable $\mid \kappa B \alpha$ protein that is not degraded after IL-1 $\beta$ stimulation (18) and keeps most of the NF-KB sequestered in the cytoplasm of these cells (Figure 4C).

Finally, using our procedure we were able to supply evidence for interaction of MCPIP1 protein with IL6 mRNA in cells. To detect this interaction, we transiently transfected HeLa cells with an MCPIP1-MycHis expression vector and detected MCPIP1 protein using anti-c-myc antibodies. IL6 mRNA was imaged using the smRNA FISH probe blend. We were able to clearly see co-localization of the MCPIP1 protein with the IL6 transcript (Figure 4D, white arrows). These observations further reinforce the data presented by Mino et al. showing binding of MCPIP1 to IL-6 mRNA and degradation of this transcript (19). An intensity line profile illustrates co-localization observed in the boxed area in more detail (Figure 4, E and F). To quantify co-localization and to generate an iso-colocalization surface, we correlated both imaging channels pixel-wise and calculated the Pearson coefficient (using Huygens Colocalization Analyzer and Surface Renderer, SVI) (Figure 4F). Even more pronounced co-localization was observed when a mutant, RNaseinactive form of the MCPIP1 protein with intact RNA binding properties (MCPIP1 D141A) was used (Supplementary Figure S7). The functionality of ectopically expressed MCPIP1-MycHis protein was verified using a luciferase reporter gene assay (Figures 4G). HeLa cells were transfected with plasmids containing the luciferase coding sequence with an attached IL-6 3'-UTR (pLuc-IL6-3’UTR). Overexpression of MCPIP1 resulted in a decrease of luciferase activity in the samples co-transfected with pLuc-IL6-3'UTR. The IL-6 3'UTR contains a conserved element with a stem-loop structure important for destabilization of the IL-6 transcript by MCPIP1. As expected, deletion of this element abolished the regulation of IL-6 3'UTR-containing transcripts by MCPIP1. Overexpression of MCPIP1 did not affect luciferase activity in samples co-transfected with a plasmid containing the luciferase coding sequence with the IL-6 $3^{\prime} U T R$ without the conserved element (pLuc-IL63'UTR $\triangle C E$ ). MCPIP1 also did not affect luciferase activity in samples co-transfected with control (empty) plasmid containing the luciferase coding sequence with no $3^{\prime}$ UTR added (pmirGLO). The presence of MCPIP1-MycHis protein in all analyzed specimens was verified by Western blot analysis revealing a single band of the predicted size of $70 \mathrm{kDa}$ (Figure 4H).

The possibility of combining IF and smRNA FISH analysis for a single specimen gives researchers a powerful tool for studying interactions of proteins with transcripts. The protocol described here enables simultaneous quantitative and spatial analysis of protein-RNA complexes in single cells. The need for single-cell transcriptome analysis was shown recently by Buettner et al. (20). Using single-cell RNA sequencing data from mouse embryonic stem cells (mESCs) with known cell cycle stages, they revealed two distinct cell subpopulations distinguished by their degree of differentiation. Thus, the protocol presented in this report can be useful in projects focusing on transcript turnover, translation, and single-cell transcriptome analysis, especially when heterogeneous cell populations are used.

\section{Author contributions}

J.K. and M.W. conceived the procedure, designed and performed the experiments, and analyzed the data. The manuscript 
was written by J.K., M.W., and A.K. All authors read and approved the manuscript.

\section{Acknowledgments}

The authors gratefully acknowledge Z. Madeja, E. Zimolag, D. Ryszawy ,and S. Lasota (Department of Cell Biology, Faculty of Biochemistry, Biophysics, and Biotechnology, Jagiellonian University, Cracow, Poland) for use of their microscope and invaluable advice. J.K. thanks P. M. Krawczyk, J.A. Aten, and J. Stap (Department of Cell Biology and Histology, Academic Medical Center, University of Amsterdam, Amsterdam, The Netherlands) for showing the way. This work was funded by the National Science Centre, Poland (NCN, Preludium Funding scheme, project number 2012/07/N/NZ1/00007 to J.K., M.W., and A.K.). J.K. and M.W. are PhD students supervised by A.K. The Faculty of Biochemistry, Biophysics and Biotechnology of Jagiellonian University is a partner of the Leading National Research Center (KNOW) supported by the Ministry of Science and Higher Education. This work was supported by the Foundation for Polish Science-PARENT-BRIDGE Programme co-financed by the European Union within European Regional Development (to A.K.).

\section{Competing interests}

The authors declare no competing interests.

\section{References}

1. Femino, A.M., F.S. Fay, K. Fogarty, and R.H. Singer. 1998. Visualization of single RNA transcripts in situ. Science 280:585-590.

2. Raj,A., P. van den Bogaard, S.A. Rifkin, A.van Oudenaarden, and S. Tyagi. 2008. Imaging individual mRNA molecules using multiple singly labeled probes. Nat. Methods 5:877-879,

3. Hocine, S., P. Raymond, D. Zenklusen, J.A. Chao, and R.H. Singer. 2013. Single-molecule analysis of gene expression using two-color RNA labeling in live yeast. Nat. Methods 10:119-121.

4. Chomczynski, P. and N. Sacchi. 2006. The single-step method of RNA isolation by acid guanidinium thiocyanate-phenol-chloroform extraction: twenty-something years on. Nat. Protoc. 1:581585.

5. Sambrook, J. and D.W. Russell. 2001. Molecular Cloning: A Laboratory Manual, 3rd ed, Cold Spring Harbor Laboratory Press, Cold Spring Harbor, NY.

6. Livak, K.J. and T.D. Schmittgen. 2001. Analysis of relative gene expression data using real-time quantitative PCR and the 2(-Delta Delta $\mathrm{C}(\mathrm{T})$ ) method. Methods 25:402-408.

7. Matsushita, K., O. Takeuchi, D.M. Standley, Y. Kumagai, T. Kawagoe, T. Miyake, T. Satoh, H. Kato, et al. 2009. Zc3h12a is an RNase essential for controlling immune responses by regulating mRNA decay. Nature 458:1185-1190.
8. Kasza, A., P. Wyrzykowska, I. Horwacik, P. Tymoszuk, D. Mizgalska, K. Palmer, H. Rokita, A.D. Sharrocks, and J. Jura. 2010. Transcription factors Elk-1 and SRF are engaged in IL1-dependent regulation of $\mathrm{ZC} 3 \mathrm{H} 12 \mathrm{~A}$ expression. BMC Mol. Biol. 11:14.

9. Yu, J.H., W.H. Yang, T. Gulick, K.D. Bloch, and D.B. Bloch. 2005. Ge-1 is a central component of the mammalian cytoplasmic mRNA processing body. RNA 11:1795-1802.

10. Caruccio, L., K. Byrne, J. Procter, and D. Stroncek. 2002. A novel method using formamide for the elution of antibodies from erythrocytes. Vox Sang. 83:63-69.

11. Shih, J.D., Z. Waks, N. Kedersha, and P.A. Silver. 2011. Visualization of single mRNAs reveals temporal association of proteins with microRNA-regulated mRNA. Nucleic Acids Res. 39:7740-7749.

12. Grünwald, D., R.H. Singer, and K. Czaplinski. 2008. Cell biology of mRNA decay. Methods Enzymol. 448:553-577.

13. Gonzalez, N., J. Wiggs, and M.J. Chamberlin. 1977. A simple procedure for resolution of Escherichia coli RNA polymerase holoenzyme from core polymerase. Arch. Biochem. Biophys. 182:404-408.

14. Toledano, H., C. D'Alterio, M. Loza-Coll, and D.L. Jones. 2012. Dual fluorescence detection of protein and RNA in Drosophila tissues. Nat. Protoc. 7:1808-1817.

15. Nakamura, K.C., H. Kameda, Y. Koshimizu, Y. Yanagawa, and T. Kaneko. 2008. Production and histological application of affinity-purified antibodies to heat-denatured green fluorescent protein. J. Histochem. Cytochem. 56:647-657.

16. Brown, K., S. Gerstberger, L. Carlson, G. Franzoso, and U. Siebenlist. 1995. Control of I kappa B-alpha proteolysis by site-specific, signalinduced phosphorylation. Science 267:14851488.

17. Skalniak, L., D. Mizgalska, A. Zarebski, P. Wyrzykowska, A. Koj, and J. Jura. 2009. Regulatory feedback loop between NF-kappaB and MCP-1-induced protein 1 RNase. FEBS J. 276:5892-5905.

18. Van Antwerp, D.J., S.J. Martin, T. Kafri, D.R. Green, and I.M. Verma. 1996. Suppression of TNF-alpha-induced apoptosis by NF-kappaB. Science 274:787-789.

19. Mino, T., Y. Murakawa, A. Fukao, A. Vandenbon, H.H. Wessels, D. Ori, T. Uehata, S. Tartey, et al. 2015. Regnase-1 and Roquin Regulate a Common Element in Inflammatory mRNAs by Spatiotemporally Distinct Mechanisms. Cell 161:1058-1073.

20. Buettner, F., K.N. Natarajan, F.P. Casale, V. Proserpio, A. Scialdone, F.J. Theis, S.A. Teichmann, J.C. Marioni, and O. Stegle. 2015. Computational analysis of cell-to-cell heterogeneity in single-cell RNA-sequencing data reveals hidden subpopulations of cells. Nat. Biotechnol. 33:155-160.

Received 06 May 2015; accepted 28 July 2015.

Address correspondence to Jakub Kochan, Department of Cell Biochemistry, Faculty of Biochemistry, Biophysics, and Biotechnology, Jagiellonian University, Cracow, Poland. E-mail:kochan.jakub@gmail.com

To purchase reprints of this article, contact: biotechniques@fosterprinting.com
This ad won't work.

Our antibodies will.

For really good antibodies, visit bethyl.com/trialsize

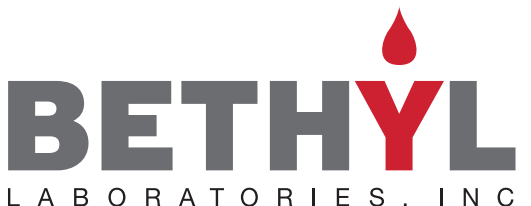

